\title{
Curvas de Distribuição de Dados sobre Germinação do Feijão: Uso para Estudo sobre o Formatado das Curvas da Pandemia de Covid-19
}

\section{Data Distribution Curves About the Bean Germination: Use for Studying the Shape of the Covid-19 Pandemic Curves}

\author{
Raul G. B. Mendes ${ }^{1,2}$ \\ ${ }^{1}$ Colégio Tiradentes da Polícia Militar de Minas Gerais, CTPM-MG, São João del Rei - MG, \\ Brasil \\ ${ }^{2}$ Departamento de Ciências Naturais, Universidade Federal de São João del Rei, São João \\ del Rei - MG, Brasil
}

\begin{abstract}
Resumo
Este artigo descreve o uso de uma práticas experimental de fácil reprodução, a germinação de grãos de feijão, para a obtenção e análise comparativa entre grupos de dados experimentais obtidos de forma aleatória e sistematizada por alunos do ensino médio da rede pública. Estes grupos de dados foram interpretados através de curvas de distribuição de dados em forma de sino (distribuição normal) e utilizados como referência comparativa para a interpretação de padrões de curvas de contaminados na pandemia por Covid-19. Ao final, o ganho em aprendizagem pelos alunos foi verificado através da aplicação de questionário com questões discursivas.
\end{abstract}

Palavras-chave: Física Experimental. Curva de distribuição de dados. Covid-19. Ensino Médio. 


\begin{abstract}
This article describes the use of an easily reproducible experimental practice, the germination of beans, to obtain and comparative analysis between groups of experimental data obtained by random and systematized ways by high school students in public schools. These data groups were interpreted using curves of data distribution like bell curve (normal distribution) and used as a comparative reference to interprete curve's patterns of the contaminated in the Covid-19 pandemic. At the end, the learning gain by the students was verified through the application of a questionnaire with discursive questions.
\end{abstract}

Keywords: Experimental Physics, Curve of data distribution, Covid-19, High School

\title{
I. INTRODUÇãO
}

A percepção de avanços tecnológicos ocorridos ao longo da recente evolução humana, como a expansão das navegações, a Guerra Fria e a corrida tecnológica pelo 5G, constantemente trazem à tona discussões sobre novas diretrizes de ensino de ciências, as quais, através de modernas e atraentes formas de ensino, garantam a permanente renovação de profissionais com perfil acadêmico/científico (PEREIRA, 2017; TRAVERS, 1973; BLOSSER, 1980). Dado isso, a importância da inserção de práticas experimentais no ensino de ciências naturais, principalmente na física, é tema amplamente estudado em artigos e periódicos nacionais e internacionais, dissertações de mestrado e teses de doutorado (GRANDINI, 2004; BLOSSER, 1988). Desde o final do século XIX o uso de práticas experimentais no ensino de temáticas relacionadas às áreas da ciências naturais têm sido utilizadas concomitantemente com a transmissão do conhecimento teórico (FAY, 1931). Decorrência da pluralidade de habilidades e limitações encontradas em uma sala de aula, o uso de práticas interdisciplinares apresenta-se como uma atraente alternativa para um contato científico mais amplo entre os alunos.

Com a obrigatoriedade de se manter isolamentos sociais durante a pandemia de Covid-19 e, consequentemente, a necessidade de se adequar o ensino de física experimental à nova realidade de ensino remoto (TAKAHASHI, 2011; SÉRÉ, 2003; ALVES, 2020; VILELA, 2019) as práticas de ensino de física convencionalmente dadas em espaço físicos apropriados de laboratórios tiveram que ser adaptadas/trocadas para serem reproduzidas em ambiente doméstico. Outro ponto importante, é que estas práticas, caso reproduzidas pelos alunos em suas respectivas casas, não contariam com a presença do professor responsável. Motivado por esta nova condição de ensino, este artigo apresenta uma prática de ensino de física interdisciplinar, a qual insere o aluno no tema da distribuição de dados e da análise de curvas de distribuição através de um simples experimento de germinação de grãos de feijão. Como interdisciplinariedade neste trabalho entende-se como a possibilidade de inserir os alunos no contexto do estudo de curvas de distribuição de dados (conhecimento matemático comumente aplicado à física experimental), ao mesmo tempo em que têm de compreender as necessidades biológicas de uma planta em seu estágio de germinação (biologia). Em seguida, 
o aluno é conduzido à interpretação de padrões de curvas de contaminados da pandemia de Covid-19 através de comparação com os formatos das curvas obtidas experimentalmente. Ao final, a viabilidade da aplicação desta prática de ensino é discutida através da análise de um conjunto de respostas dadas pelos próprios alunos a perguntas específicas sobre temas relacionados ao trabalho desenvolvido por eles, tais como: análise de curvas de distribuição de dados, respeito aos métodos experimentais e análise de dados estatísticos.

\section{CONSIDERAÇões Metodológicas}

A atividade didática descrita neste trabalho consistiu na investigação e compreensão de curvas de distribuição de dados estatísticos obtidos experimentalmente e de padrões teóricos. Através de reproduções sucessivas de uma prática experimental sobre germinação de grãos de feijão, os alunos foram avaliados quanto às suas capacidades de interpretar, comparativamente, os dados experimentais obtidos com curvas de padrões teóricos sobre a contaminação por COVID-19 em populações. Toda a atividade foi aplicada aos alunos em aulas remotas de laboratório de física, ocorridas ao longo do período de isolamento social do ano de 2020 devido a pandemia de Covid-19.

Inicialmente, cada aluno participante da atividade teve que realizar o experimento de germinação do feijão em sua respectiva residência. Todos os materiais experimentais utilizados para a execução da prática foram itens domésticos comuns e de fácil acesso a todos os alunos. As instruções necessárias para a realização da prática foram passadas aos alunos através de uma vídeo aula preliminar de 50 minutos de duração e um roteiro experimental. Ao longo do período de execução da prática os alunos foram assistidos pelo professor da disciplina por vídeo aulas semanais e, diariamente, por trocas de mensagens por webchat. Em seguida, foi realizada a interpretação e discussão dos dados experimentais obtidos por todos os alunos, onde os dados experimentais foram divididos em dois grupos e plotados em forma de gráficos de barra (Fig-1, seção de Resultados e Discussões).

Em um segundo momento, cerca de cem dias após terem completado a etapa anterior, com auxílio do professor, os mesmos alunos responderam a algumas questões discursivas relacionadas à pratica experimental e à associação dos resultados obtidos com padrões de curvas de contaminação por Covid-19. Ao final, o grau de aprendizagem dos alunos com esta atividade foi avaliado através da análise das respostas dadas pelos alunos.

\section{II.1. Participantes}

A prática experimental foi realizada com alunos dos três anos do ensino médio, de uma escola cívico-militar da rede pública estadual de ensino do estado de Minas Gerais, na cidade de de São João del Rei. Ao total, 86 alunos participaram da atividade experimental, com idades entre 14 e 18 anos. ${ }^{1}$

\footnotetext{
${ }^{1}$ A escolha por trabalhar com alunos dos três anos do ensino médio decorreu, unicamente, pela limitação na quantidade de alunos matriculados em cada um destes anos escolares na escola em questão. Visto que o trabalho trata-se de uma análise de distribuição de dados, um baixo número de alunos participantes poderia inviabilizar a análise estatística dos dados coletados.
} 


\section{II.2. Atividade Desenvolvida}

A introdução à atividade didática ocorreu pela discussão com os alunos sobre a importância de se respeitar critérios para a reprodução de práticas experimentais, a fim de se garantir uma fidelidade mínima necessária para a comparação entre resultados obtidos em práticas experimentais realizadas por pessoas diferentes e em locais distintos. Em seguida, discutiu-se com os alunos a relevância de se interpretar estes dados como uma composição de uma distribuição estatística. Discutiu-se, também, o conceito de correlação entre dados experimentais e de como esta correlação entre os dados, sendo alta ou baixa, deve influenciar no formato mais concentrado ou mais disperso da curva de distribuição destes dados. O próximo passo foi estabelecer junto com os alunos um método padronizado para que eles pudessem germinar grãos de feijão em algodão, em suas respectivas residências, a fim de se ter germinações o mais parecidas possível, ocorrendo em locais distintos e por alunos diferentes. Neste padrão de germinação foi definido o tipo de feijão, o tamanho do pedaço de algodão a ser utilizado, o recipiente no qual o grão e o algodão seriam inseridos, a taxa de regagem e quantidade de água regada e a forma de exposição à luz do Sol. Para a consolidação de toda esta primeira etapa instrutiva foi utilizado um horário de aula (50 min.) para cada uma das três turmas de alunos, $1^{\circ}, 2^{\circ}$ e $3^{\circ}$ anos do ensino médio. Um roteiro experimental descrevendo a forma como a prática deveria ser realizada foi passado aos alunos.

A prática experimental consistiu em cada aluno plantar dez grãos de feijão em algodão em suas respectivas residências. Cinco destes grãos de feijão compuseram o que ficou

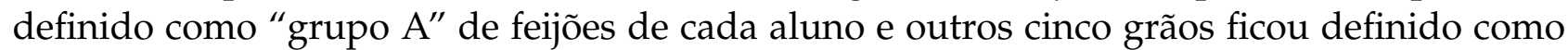
"grupo B". Assim, se considerarmos $\mathbf{n}$ alunos reproduzindo a prática experimental, teríamos 5n grãos pertencentes ao grupo $\mathrm{A}_{\text {total }}$ e outros 5 n grãos pertencentes ao grupo $\mathrm{B}_{\text {total }}$. Para os plantios de cada grupo A pediu-se que os alunos respeitassem ao máximo o padrão preestabelecido de plantio, para que, assim, as correlações entre os processos de germinação dos grãos pertencentes ao $\mathrm{A}_{\text {total }}$ fossem as maiores possíveis. Já para os grãos dos grupos $\mathrm{B}$ não foi exigido nenhum padrão nos plantios, os feijões poderiam ser de tipos diferentes, $\mathrm{O}$ tamanho dos algodões usados, a taxa de regagem e os locais onde ocorreriam as germinações deveriam ser escolhidos de forma aleatória por cada aluno. Neste segundo grupo buscou-se não estabelecer critérios entra as suas $\mathbf{5 n}$ germinações, a fim de se diminuir correlações entre os processos de plantio, porém sem que os alunos deixassem de prezar pela efetiva germinação de cada grão. Neste grupo os grãos deveriam germinar e se desenvolver, mas sem um padrão único induzido pelos alunos, estes desenvolvimentos dos grãos deveriam ocorrer de forma mais individualizada, ou seja, aleatória.

Com a evolução da germinação de cada grão de feijão, este deveria apresentar suas folhas. Assim, juntamente com os alunos, previamente, definiu-se como evento experimental a ser investigado a aparição da primeira folha de cada grão de feijão.

Iniciando a prática experimental todos ao mesmo tempo, os alunos tiveram que plantar seus dez grãos de feijão, cinco do grupo A mais cinco do grupo B, em suas respectivas residências. Após o plantio, os alunos acompanharam os processos de germinação ao longo de vinte e um dias. Os alunos tiveram que anotar quantos dias, a partir do início da prática, cada um de seus feijões levou para apresentar sua primeira folha. 
Ao final da prática experimental, com ajuda do professor, os dados de todos os alunos foram reunidos em grupo $A_{\text {total }}$ e grupo $B_{\text {total }}$ e interpretados como curvas de distribuição, número de eventos observados (quantidade de aparição da primeira folha de feijão) em função da quantidade de dias. A interpretação dos dados foi embasada nas condições de germinação de cada um dos dois grupos, condição com alta correlação entre as germinações (grupo $\mathrm{A}_{\text {total }}$ ) e condição com baixa correlação entre as germinações (grupo $\mathrm{B}_{\text {total}}$ ). A análise dos dados foi desenvolvida comparando os resultados obtidos com os padrões de curvas de contaminação da Covid-19 com e sem isolamento social.

Após um período de, aproximadamente, cem dias após a conclusão da prática experimental e discussão dos dados, os alunos foram avaliados. Através das respostas dadas pelos alunos a um conjunto de questões discursivas procurou-se mensurar a capacidade obtida pelos alunos para analisar curvas de distribuição de dados, sobre a importância de se respeitar métodos experimentais e análise de dados estatísticos.

\section{Resultados E Discussões}

A Fig-1 exibe as distribuições por histogramas dos resultados encontrados por todos os alunos, para as germinações relacionadas aos grupos $\mathrm{A}_{\text {total }}$ e $\mathrm{B}_{\text {total }}$. Com os dados plotados exibindo a porcentagem de eventos de germinação ocorridos a cada dia, em função dos dias a partir do início do experimento, curvas em forma de sino (distribuições gaussianas) foram ajustadas a cada um dos dois grupos de dados experimentais pelo próprio professor, curvas em forma de sino em linha contínua e em linha tracejada ${ }^{2}$.

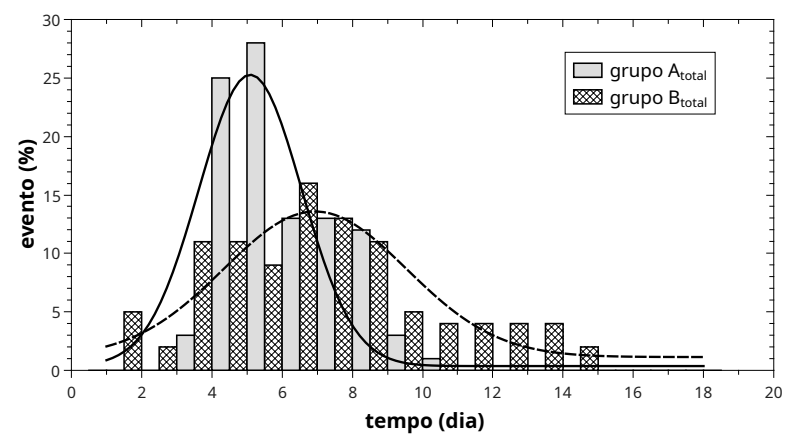

Figura 1: Histogramas da porcentagem de observação da aparição da primeira folha de germinação (\% de

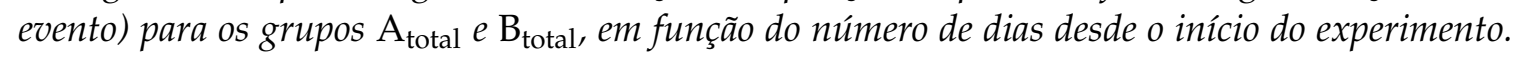

A Fig-2 apresenta dois padrões teóricos de curvas de duas situações distintas na contaminação pelo vírus Sars-Cov-2, as situações onde ocorre o isolamento social (com intervenção do estado) e onde não ocorre isolamento social (sem intervenção do estado).

Abaixo, são apresentadas as questões que foram passadas aos alunos para que eles respondessem após um período de, aproximadamente, cem dias após a realização da prática experimental. Através destas questões buscou-se avaliar a capacidade dos alunos de

\footnotetext{
${ }^{2}$ Para a plotagem em gráficos de barra e ajuste das curvas gaussianas usou-se o software de distribuição gratuita Qtiplot
} 


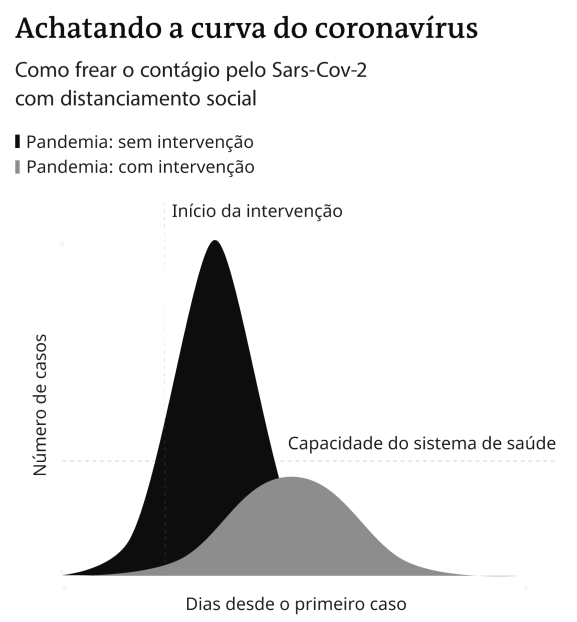

Figura 2: Curva teórica para a distribuição do número de casos de infectados por COVID-19 em função dos dias, para as condições sem isolamento social (sem intervenção) e com isolamento social (com intervenção).(OS NÚMEROS, 2020)

Fonte: $O$ autor.

interpretarem curvas de distribuição de dados estatísticos através do formato das curvas e a capacidade de estimarem o quão correlacionados devem ser os eventos observados em cada conjunto de dados analisado. Além disso, buscou-se avaliar a compreensão dos alunos quanto à preservação de padrões para a reprodução experimental e coleta de dados. Juntamente com cada uma das questões apresentadas aos alunos, são apresentados alguns trechos extraídos de suas respostas.

Questão-1

Tomemos como comparação a conjuntura atual, com a pandemia causada pelo vírus SarsCov-2, da família coronavírus e responsável pela doença Covid-19. Supondo que todos os indivíduos de uma determinada população em algum momento ao longo do ano de 2020 serão infectados pelo Sars-Cov-2, então a Fig-2 nos mostra duas situações distintas para a quantidade de infectados (Número de casos) em função do tempo gasto para se infectar (Dias desde o primeiro caso). Como podemos relacionar as formas das curvas experimentais obtidas para os grupos $A_{\text {total }}$ e $B_{\text {total}}$, Fig-1, com as formas das curvas da pandemia "sem intervenção" e "com intervenção", apresentadas na Fig-2?

Nesta questão esperou-se que os alunos fossem capazes de assemelhar a curva $A_{\text {total }}$ da Fig-1 com a curva Sem Intervenção da Fig-2, por suas formas mais estreitas, as quais representam maior concentração de eventos em torno de um instante específico. Similarmente, que também fossem capazes de assemelhar a curva $B_{\text {total }}$ com a curva Com Intervenção, por apresentarem uma distribuição mais dispersas ao longo do tempo. Para tal questionamento, destaca-se as respostas obtidas:

"É notório que o grupo A total (gráfico-1) e o grupo Pandemia: sem intervenção (gráfico2) apresentam curvas elevadas, simbolizando que o seu desenvolvimento foi mais rápido em relação as curvas menores, que são do grupo B total e do grupo Pandemia: com intervenção, 
os quais demoraram se desenvolver devido a interferência em ambas situações."

"A curva do grupo A corresponde à curva da pandemia "sem intervenção", enquanto a curva do grupo B corresponde à curva da pandemia "com intervenção"."

"Podemos relacionar as curvas experimentais do grupo A com as curvas da pandemia sem intervenção, isso porque a ação de brotação ocorre praticamente ao mesmo tempo em todos os feijões. Já o grupo B com as curvas da pandemia com intervenção, por ter mais distinção em relação ao tempo de nascimento da folha. "

Dos trechos de resposta acima, observa-se que os alunos conseguiram, através da comparação dos perfis das curvas, fazer as corretas associações entre as distribuições dos dados, como distribuições mais concentrada ou mais dispersas dos eventos ocorridos.

Já na questão abaixo buscou-se avaliar o entendimento do aluno de causa e efeito sobre o amortecimento das curvas, a compreensão sobre a existência de correlação entre os eventos de uma mesma amostragem e como esta correlação influencia no formato da curva de distribuição, deixando-a mais concentrada quando a correlação entre os eventos é alta ou resultando em uma curva mais dispersa quando a correlação é baixa.

\section{Questão-2}

Se definirmos como eventos probabilísticos o ato de brotar uma folha de feijão e o ato de se infectar pelo Sars-Cov-2, baseando-se na discussão feita a cerca dos resultados experimentais que obtivemos das germinações, em qual das duas curvas da pandemia apresentadas na Fig-2 observa-se maior correlação entre os atos de se infectar pelo Sars-Cov-2 em uma população? Quais as características das curvas que evidenciam isto?

“A curva da pandemia sem intervenção, que usando a relação da folha de feijão e do ato de se infectar, tem relação direta com a curva A total por ambos mostrarem o surgimento de vários "atos" de uma só vez"

"Na curva sem intervenção há uma súbita contaminação, já na com intervenção a contaminação é mais duradoura, porém mais controlada. Isto é evidenciado pela altura e prolongamento da curva."

"Na curva da pandemia "sem intervenção", pois muitos indivíduos serão infectados em um curto período de tempo, o que podemos perceber pelo formato da curva: alta (eixo y, que representa o número de casos) e de base estreita (eixo x, que representa o tempo)."

As respostas demonstram que os alunos compreendem bem como ações externas podem aumentar ou diminuir a correlação entre dados de uma amostragem e como o nível desta correlação existente influencia no formato da curva de distribuição de dados.

“[...] a curva A. Isso porque, de acordo com a análise das curvas, ela aumenta drasticamente com o passar do tempo, como uma função exponencial."

"Na curva de A total é possível observar maior relação entre os atos de se infectar pelo Sars-Cov-2, pois essa curva se eleva em um pouco espaço de tempo, o que significa que grande número de pessoas se infectariam pelo Sars-Cov-2 em um tempo curto"

Nestes dois últimos trechos os alunos confundiram o nome da curva, responderam citando a curva da Fig-1, contudo demonstraram compreensão do conceito esperado, conse- 
guiram associar o conceito de correlação entre eventos estatísticos com o formato da curva de distribuição de dados. Trechos como, "o que significa que grande número de pessoas se infectariam pelo Sars-Cov-2 em um tempo curto" aponta o alcance de um dos principais objetivos deste trabalho, possibilitar ao aluno a compreensão da pandemia pelo Sars-Cov-2 como um fenômeno estatístico e, assim, interpretá-lo como tal.

\section{Questão-3}

Qual a conclusão que se pode tirar deste experimento, sobre:

a) A importância de se respeitar padrões de reprodução experimental para a reprodução experimental para a interpretação do dados coletados?

“... garante que a observação de um determinado experimento tenha validade para a definição ou constatação de uma teoria científica."

"Sem os padrões de reprodução experimental seria impossível interpretar os dados obtidos no experimento"

“... com ele práticas e experimentos ganham cunho verídico e seus resultados podem comprovar teorias científicas."

"Através de métodos científicos obtemos respostas e amostras de teorias que antes estavam somente no papel e não eram palpáveis. Após a experimentação,pode-se constatar os resultados que serão utilizados para as áreas necessárias, auxiliando de várias maneiras em pesquisas importantes como as da produção da vacina contra o COVID-19."

A respostas apresentadas acima pelos alunos evidencia a compreensão destes da necessidade em se respeitar metodologias de trabalho a fim de se garantir resultados que possam ser interpretados como um conjunto de dados.

b) A importância de se poder fazer um trabalho experimental em grupo, buscando-se maior quantidade de observações a cerca de uma questão a ser investigada?

“Sua importância está relacionada ao modo de observar de cada pessoa, que por ser diferente, permite a visualização de novas coisas e lados que não poderíamos observar sozinhos. Além disso, em um trabalho em grupo, podemos realizar um mesmo experimento por diversas pessoas, o que permite evidenciar a diferença na prática de cada um."

"Haverá mais resultados para a análise, de modo que a pesquisa seja mais bem desenvolvida, uma vez que comprovará se o resultado obtido é um caso particular ou geral."

"Ao realizarmos um trabalho experimental em grupo, temos mais resultados para analisar, sendo possível uma melhor análise mais correta e detalhada acerca do que está sendo estudado."

"A importância de se fazer um trabalho experimental em grupo está no fato que se terá diferentes pontos de vistas e a possibilidade de maior número de observações, com a possibilidade de um resultado mais correto e bem analisado."

"Em um trabalho experimental pode haver diferentes tipos de resultados, o que pode indicar erros, diferentes processos com resultados semelhantes, ou até mesmo resultados mais satisfatórios, tal fato ocorreu durante a experiência com os feijões, na qual nem todos os alunos que a realizaram tiveram o mesmo resultado e outros tiveram resultados mais 
satisfatórios comparados aos demais. [...] o trabalho experimental em grupo proporciona uma diversidade de opiniões e visões sobre o mesmo experimento, contribuindo para um maior êxito da mesma experiência ou de posteriores."

"A importância deste método é, entre outras, realizar experimentos onde não somente uma amostra é analisada, mas também diversas outras que podem ou não seguirem de maneiras iguais, dependendo da forma como são administradas. Logo, nota-se que maneiras diferentes de se realizar um experimento,trazem resultados diferentes,o que pode contribuir para diversas pesquisas, verificando-se qual o melhor caminho a ser seguido."

Para esta última questão procurou-se constatar se os alunos realmente compreenderam a importância de se obter várias medidas de um mesmo experimento para uma análise mais apurada do experimento. Além desta constatação, pôde-se observar a incorporação por parte dos alunos quanto aos benefícios de se trabalhar em grupo, dada a possibilidade de obtenção de um maior número de medidas de uma mesma prática experimental e, por conseguinte, menor influência de erros experimentais nos valores encontrados.

\section{Conclusão}

Neste artigo foi apresentada a aplicação, e o consequente desenvolvimento didático, de uma prática experimental interdisciplinar aplicada em aulas remotas de laboratório de física para alunos do ensino médio da rede pública de ensino do estado de Minas Gerais. Por a prática basear-se na reprodução sismetatizada de um experimento de fácil reprodução, germinação de grãos de feijão em algodão, ela mostrou-se como uma rica proposta experimental a ser desenvolvida durante situações de ensino remoto. $\mathrm{O}$ uso dos resultados experimentais obtidos, e de suas respectivas interpretações, possibilitaram a extrapolação do experimento de germinação para o contexto pandêmico, vivido pelos próprios alunos devido a pandemia de Covid-19. A possibilidade de vislumbrar padrões de curvas de contaminados em uma pandemia como curvas de distribuição de dados, similares aos obtidos por um simples experimento de germinação de grãos de feijão, possibilitou aos alunos uma rica experiência didática interdisciplinar, focada na interpretação e compreensão de curvas estatísticas relacionados ao contexto de pandemia mundial, na qual os alunos encontravam-se inseridos ao longo do ano de 2020.

\section{Agradecimentos}

O autor agradece ao professor Alessandro Damásio Trani Gomes (Departamento de Ciências Naturais da Universidade Federal de São João del Rei) por conselhos e correções na escrita do texto.

\section{REFERÊNCIAS}

PEREIRA, M. V. e AMARAL, M. C. M. (2017) Caderno Brasileiro de Ensino de Física, 34, n.1, 265. 
TRAVERS, R. M. W. (1973) in: Second handbook of research on teaching: A project of the American Educational Research Association, Rand McNally.

BLOSSER, P. E. (1980) in: A Critical Review of the Role of the Laboratory in Science Teaching, ed. ERIC.

GRANDINI, N. A., GRANDINI, C. R. (2004) Revista Brasileira de Ensino de Física, 26, n.3, 251.

BLOSSER, P. E. (1988) Caderno Brasileiro de Ensino de Física, 5, n.2, 74.

FAY, P. J. (1931) Journal of Chemical Education, 8, n.8, 1533.

TAKAHASHI, E. K., CARDOSO, D. C. (2011) Revista Brasileira de Pesquisa em Educação em Ciências, 11, n.3, 185.

SÉRÉ, M. G., COELHO, S. M., NUNES, A. D. (2003) Caderno Brasileiro de Ensino de Física, 20, n.1, 30.

ALVES, J. N. et. al. (2020) Revista Thema, 18, 184.

VILELA, D. C. et. al. (2019) Revista Brasileira de Ensino de Física, 41, n.4, 20190041.

OS NÚMEROS sobre a pandemia de coronavírus. [S. 1.], 20 mar. 2020. Disponível em:

https://www.dw.com/pt-br/os-n\%C3\%BAmeros-sobre-a-pandemia-de-coronav\%C3\%ADrus/a52848559. Acesso em: 15 set. 2020. 